\title{
On a general bilinear functional equation
}

\author{
Anna Bahyrycz And Justyna SikorskA (D
}

Dedicated to Professor Ludwig Reich on the occasion of his 80th birthday.

Abstract. Let $X, Y$ be linear spaces over a field $\mathbb{K}$. Assume that $f: X^{2} \rightarrow Y$ satisfies the general linear equation with respect to the first and with respect to the second variables, that is,

$$
\left\{\begin{array}{l}
f\left(a_{1} x_{1}+a_{2} x_{2}, y\right)=A_{1} f\left(x_{1}, y\right)+A_{2} f\left(x_{2}, y\right) \\
f\left(x, b_{1} y_{1}+b_{2} y_{2}\right)=B_{1} f\left(x, y_{1}\right)+B_{2} f\left(x, y_{2}\right)
\end{array}\right.
$$

for all $x, x_{i}, y, y_{i} \in X$ and with $a_{i}, b_{i} \in \mathbb{K} \backslash\{0\}, A_{i}, B_{i} \in \mathbb{K}(i \in\{1,2\})$. It is easy to see that such a function satisfies the functional equation

$$
\begin{aligned}
f\left(a_{1} x_{1}+a_{2} x_{2}, b_{1} y_{1}+b_{2} y_{2}\right)= & C_{1} f\left(x_{1}, y_{1}\right)+C_{2} f\left(x_{1}, y_{2}\right) \\
& +C_{3} f\left(x_{2}, y_{1}\right)+C_{4} f\left(x_{2}, y_{2}\right),
\end{aligned}
$$

for all $x_{i}, y_{i} \in X(i \in\{1,2\})$, where $C_{1}:=A_{1} B_{1}, C_{2}:=A_{1} B_{2}, C_{3}:=A_{2} B_{1}, C_{4}:=A_{2} B_{2}$. We describe the form of solutions and study relations between $(*)$ and $(* *)$.

Mathematics Subject Classification. 39B52, 39B72, 15A06, 12 F05.

Keywords. Linear equation, Additive function, Biadditive function, Hamel basis, Field extension, Algebraic dependence.

\section{Introduction}

General linear functional equations have been studied for years (see, e.g., [1-4, $6,10])$. In the paper, we shall study their counterpart for two-variable functions.

Let $X, Y$ be linear spaces over a field $\mathbb{K}$ and $f: X^{2} \rightarrow Y$. For some fixed $a_{i}, b_{i} \in \mathbb{K} \backslash\{0\}=: \mathbb{K}^{*}, A_{i}, B_{i} \in \mathbb{K}, i \in\{1,2\}$, we consider the following system

$$
\left\{\begin{array}{l}
f\left(a_{1} x_{1}+a_{2} x_{2}, y\right)=A_{1} f\left(x_{1}, y\right)+A_{2} f\left(x_{2}, y\right) \\
f\left(x, b_{1} y_{1}+b_{2} y_{2}\right)=B_{1} f\left(x, y_{1}\right)+B_{2} f\left(x, y_{2}\right)
\end{array}\right.
$$

for all $x, x_{i}, y, y_{i} \in X, i \in\{1,2\}$. 
It is easy to see that from (1) we immediately get

$$
\begin{aligned}
f\left(a_{1} x_{1}+a_{2} x_{2}, b_{1} y_{1}+b_{2} y_{2}\right)= & A_{1} B_{1} f\left(x_{1}, y_{1}\right)+A_{1} B_{2} f\left(x_{1}, y_{2}\right) \\
& +A_{2} B_{1} f\left(x_{2}, y_{1}\right)+A_{2} B_{2} f\left(x_{2}, y_{2}\right),
\end{aligned}
$$

which leads to a more general equation

$$
\begin{aligned}
f\left(a_{1} x_{1}+a_{2} x_{2}, b_{1} y_{1}+b_{2} y_{2}\right)= & C_{1} f\left(x_{1}, y_{1}\right)+C_{2} f\left(x_{1}, y_{2}\right) \\
& +C_{3} f\left(x_{2}, y_{1}\right)+C_{4} f\left(x_{2}, y_{2}\right)
\end{aligned}
$$

for all $x_{i}, y_{i} \in X$ and with fixed $a_{i}, b_{i} \in \mathbb{K}^{*}, C_{j} \in \mathbb{K}, i \in\{1,2\}, j \in\{1,2,3,4\}$.

In [5], Ciepliński asked about the general solution of (3). He also formulated the problem whether equation (3) (or (2)) is equivalent to system (1) (by 'equivalent' we mean here that they have the same sets of solutions), or under which assumptions on coefficients they are equivalent.

As already observed, from (1) we immediately get (3) with $C_{1}:=A_{1} B_{1}$, $C_{2}:=A_{1} B_{2}, C_{3}:=A_{2} B_{1}, C_{4}:=A_{2} B_{2}$.

It is interesting to compare the following examples.

Example 1. (a) The function $f(x, y)=x y+x+y+1, x, y \in \mathbb{R}$, satisfies (3) and (1) with $a_{i}=b_{i}=A_{i}=B_{i}=\frac{1}{2}$ and $C_{j}=\frac{1}{4}$ for $i \in\{1,2\}$, $j \in\{1,2,3,4\}$.

(b) The function $f(x, y)=x+y, x, y \in \mathbb{R}$, satisfies (3) with $a_{i}=b_{i}=2$ and $C_{j}=1$ for $i \in\{1,2\}, j \in\{1,2,3,4\}$, but does not satisfy (1) with any coefficients $A_{i}, B_{i}$.

(c) The function $f(x, y)=2 x, x, y \in \mathbb{R}$, satisfies both (3) and (1) with $a_{i}=A_{i}=2, b_{1}=1, b_{2}=3, B_{1}=\frac{1}{3}, B_{2}=\frac{2}{3}$ and $C_{j}=1$ for $i \in\{1,2\}$, $j \in\{1,2,3,4\}$ even though $A_{1} B_{1} \neq C_{1}, A_{1} B_{2} \neq C_{2}$ and so on.

From Example 1 we see, e.g., that the conditions $C_{1}=A_{1} B_{1}, C_{2}=A_{1} B_{2}$, $C_{3}=A_{2} B_{1}, C_{4}=A_{2} B_{2}$ do not guarantee the equivalence between (1) and (3). In the final part of the paper we will describe circumstances under which (3) implies (1).

Apart from this, we can formulate a problem in the language of alienation: Given (3), we ask when there exist $A_{1}, A_{2}, B_{1}, B_{2}$ such that (3) splits into two equations from (1). For details concerning the description and the general idea of the alienation phenomenon we refer the interested reader to $[7,8]$.

In the paper we restrict ourselves to the case where $\mathbb{K}$ is a field of characteristic zero. Then it is an extension of the field $\mathbb{Q}$ of the rationals.

\section{Solutions of (1)}

We start with the following 
Theorem 1. If a function $f: X^{2} \rightarrow Y$ satisfies (1) then there exist a biadditive function $g: X^{2} \rightarrow Y$, additive functions $\varphi, \psi: X \rightarrow Y$ and a constant $\delta \in Y$ such that

$$
f(x, y)=g(x, y)+\varphi(x)+\psi(y)+\delta
$$

and

$$
\begin{aligned}
g\left(a_{i} x, y\right) & =A_{i} g(x, y), \\
g\left(x, b_{i} y\right) & =B_{i} g(x, y), \\
\varphi\left(a_{i} x\right) & =A_{i} \varphi(x), \\
\psi\left(b_{i} y\right) & =B_{i} \psi(y),
\end{aligned}
$$

for all $x, y \in X$ and $i \in\{1,2\}$, and, moreover,

$$
\begin{aligned}
& \psi=0 \text { and } \delta=0, \text { whenever } A_{1}+A_{2} \neq 1 \\
& \varphi=0 \text { and } \delta=0, \text { whenever } B_{1}+B_{2} \neq 1 .
\end{aligned}
$$

Conversely, for every biadditive function $g: X^{2} \rightarrow Y$, additive functions $\varphi, \psi: X \rightarrow Y$ such that conditions (5), (6), (7) hold for all $x, y \in X$ and $i \in\{1,2\}$ and for every $\delta \in Y$ such that

$$
\delta\left(A_{1}+A_{2}-1\right)=\delta\left(B_{1}+B_{2}-1\right)=0,
$$

the function $f: X^{2} \rightarrow Y$ of the form (4) is a solution to (1).

Proof. Assume $f$ satisfies (1), that is $f$ satisfies the general linear equation with respect to the first variable and with respect to the second variable. Hence, (see, e.g., Kuczma [10, p. 383])

$$
\begin{aligned}
& f\left(x_{1}+x_{2}, y\right)=f\left(x_{1}, y\right)+f\left(x_{2}, y\right)-f(0, y), \\
& f\left(x, y_{1}+y_{2}\right)=f\left(x, y_{1}\right)+f\left(x, y_{2}\right)-f(x, 0),
\end{aligned}
$$

for all $x, x_{1}, x_{2}, y, y_{1}, y_{2} \in X$. It follows that the functions

$$
\varphi(x):=f(x, 0)-f(0,0), x \in X, \text { and } \psi(y):=f(0, y)-f(0,0), y \in X,
$$

are additive and satisfy (6) and (7), respectively. Furthermore, the function

$$
g(x, y):=f(x, y)-f(x, 0)-f(0, y)+f(0,0), \quad x, y \in X,
$$

is, by (10), biadditive and satisfies (5).

Moreover, from (1) we have

$$
\begin{aligned}
& f(0, y)=\left(A_{1}+A_{2}\right) f(0, y), \\
& f(x, 0)=\left(B_{1}+B_{2}\right) f(x, 0),
\end{aligned}
$$

for all $x, y \in X$. That is, if $A_{1}+A_{2} \neq 1$ then $f(0, y)=0$ for all $y \in X$ and whence, $\psi(y):=f(0, y)-f(0,0)$ for all $y \in Y$ and $\delta:=f(0,0)$ vanishes. Similarly, if $B_{1}+B_{2} \neq 1$ then $f(x, 0)=0$ for all $x \in X$ and $\varphi(x):=f(x, 0)-$ $f(0,0)$ for $x \in X$ and $\delta$ vanishes. Consequently, we have (8).

The proof of the converse is a direct computation. 
Directly from (1) we have the following.

Remark 1. If $A_{1}+A_{2}=1$ and $B_{1}+B_{2}=1$ then every constant function $f: X^{2} \rightarrow Y$ satisfies (1). Otherwise, $f=0$ is the only constant function satisfying (1).

For the next considerations in which we study solutions of (1) in some special cases we introduce the following conditions:

$$
\begin{gathered}
\forall_{i \in\{1,2\}} a_{i}=A_{i} \wedge \forall_{i \in\{1,2\}} b_{i}=B_{i}, \\
\forall_{i \in\{1,2\}} a_{i}=A_{i} \wedge B_{1}+B_{2}=1, \\
A_{1}+A_{2}=1 \wedge \forall_{i \in\{1,2\}} b_{i}=B_{i}, \\
A_{1}+A_{2}=1 \wedge B_{1}+B_{2}=1,
\end{gathered}
$$

and $\sim(\vartheta)$ means that condition $(\vartheta)$ fails for $\vartheta \in\{\alpha, \beta, \gamma, \delta\}$.

Corollary 1. Let $f: \mathbb{R}^{2} \rightarrow \mathbb{R}$ be a solution of (1) and assume that it satisfies any regularity condition that forces a biadditive function to be continuous. Then there exist $\alpha, \beta, \gamma, \delta \in \mathbb{R}$ such that

$$
f(x, y)=\alpha x y+\beta x+\gamma y+\delta, \quad x, y \in \mathbb{R},
$$

and moreover,

$$
\begin{aligned}
& \alpha=0, \text { whenever } \sim(\alpha) \\
& \beta=0, \text { whenever } \sim(\beta) \\
& \gamma=0, \text { whenever } \sim(\gamma) \\
& \delta=0, \text { whenever } \sim(\delta) .
\end{aligned}
$$

Proof. On account of the regularity assumption it follows that $f$ is continuous and so, by Theorem 1 any solution of (1) is of the form $f(x, y)=\alpha x y+\beta x+$ $\gamma y+\delta, x, y \in \mathbb{R}$ with some $\alpha, \beta, \gamma, \delta \in \mathbb{R}$. Therefrom we derive the following conditions

$$
\begin{array}{lll}
\alpha a_{1}=\alpha A_{1} & \alpha b_{1}=\alpha B_{1} \\
\alpha a_{2}=\alpha A_{2} & \alpha b_{2}=\alpha B_{2} \\
\beta a_{1}=\beta A_{1} & \text { and } & \beta=\beta\left(B_{1}+B_{2}\right) \\
\beta a_{2}=\beta A_{2} & \gamma b_{1}=\gamma B_{1} \\
\gamma=\gamma\left(A_{1}+A_{2}\right) & & \gamma b_{2}=\gamma B_{2} \\
\delta=\delta\left(A_{1}+A_{2}\right) & \delta=\delta\left(B_{1}+B_{2}\right),
\end{array}
$$

which lead to our assertion.

In the following results we study solutions of (1) with some rational coefficients. 
Corollary 2. Let $f: X^{2} \rightarrow Y$ satisfy (1) and let coefficients $a_{i}, b_{i}$ for $i \in\{1,2\}$ be rational. Then there exist a biadditive function $g: X^{2} \rightarrow Y$, additive functions $\varphi, \psi: X \rightarrow Y$ and a constant $\delta \in Y$ such that $f$ has the form (4), and moreover,

$$
\begin{aligned}
& g=0, \text { whenever } \sim(\alpha) \\
& \varphi=0, \text { whenever } \sim(\beta) \\
& \psi=0, \text { whenever } \sim(\gamma) \\
& \delta=0, \text { whenever } \sim(\delta) .
\end{aligned}
$$

Remark 2. By Corollaries 1 and 2 (where in Corollary $1, g(x, y):=\alpha x y$, $\varphi(x):=\beta x, \psi(y):=\gamma y$ for $x, y \in X=\mathbb{R}, Y=\mathbb{R})$, in the described special cases, it follows immediately that

- there exists a non-zero biadditive $g$ which is a solution of (1) if and only if $(\alpha)$ holds;

- there exists a non-zero additive function $\varphi$ on $X$ such that $f(x, y):=\varphi(x)$ for all $x, y \in X$ is a solution of (1) if and only if $(\beta)$ holds;

- there exists a non-zero additive function $\psi$ on $X$ such that $f(x, y):=\psi(y)$ for all $x, y \in X$ is a solution of (1) if and only if $(\gamma)$ holds;

- there exists a non-zero constant $\delta \in Y$ such that function $f(x, y) \equiv \delta$ is a solution of (1) if and only if $(\delta)$ holds (cf., Remark 1 ).

Corollary 3. Suppose system (1) has a non-constant solution $f: X^{2} \rightarrow Y$. Then

(a) if $A_{1}+A_{2} \neq 1$ and for some $i \in\{1,2\}, a_{i}$ or $A_{i}$ is rational, then $a_{i}=A_{i}$;

(b) if $B_{1}+B_{2} \neq 1$ and for some $i \in\{1,2\}, b_{i}$ or $B_{i}$ is rational, then $b_{i}=B_{i}$;

(c) if for some $i \in\{1,2\}, a_{i}$ or $A_{i}$ is rational, and for some $j \in\{1,2\}, b_{j}$ or $B_{j}$ is rational, then $a_{i}=A_{i}$ or $b_{j}=B_{j}$.

Proof. Assume $a_{1} \in \mathbb{Q}$. Then by (5) we obtain

$$
\left(a_{1}-A_{1}\right) g(x, y)=0, \quad x, y \in X,
$$

and by (6),

$$
\left(a_{1}-A_{1}\right) \varphi(x)=0, \quad x \in X .
$$

Consequently, $a_{1}=A_{1}$ or $g(x, y)=\varphi(x)=0$ for all $x, y \in X$, and $f$ depends only on the second variable. The analogous result we obtain if we assume that $a_{2} \in \mathbb{Q}$.

Assume now that $A_{1} \in \mathbb{Q}$. Then

$$
g\left(\left(a_{1}-A_{1}\right) x, y\right)=0, x, y \in X, \quad \text { and } \quad \varphi\left(\left(a_{1}-A_{1}\right) x\right)=0, \quad x \in X,
$$

and we obtain the same result as above. That is, for any $i \in\{1,2\}$,

$$
a_{i} \in \mathbb{Q} \vee A_{i} \in \mathbb{Q} \Rightarrow\left(a_{i}=A_{i} \vee \quad f(x, y)=\psi(y)+\delta, x, y \in X\right) .
$$

Analogously, we obtain

$$
b_{i} \in \mathbb{Q} \vee B_{i} \in \mathbb{Q} \Rightarrow\left(b_{i}=B_{i} \vee \quad f(x, y)=\varphi(x)+\delta, x, y \in X\right) .
$$


Items (a) and (b) follow immediately from (A), (B) and Theorem 1.

For (c), suppose that $a_{i}$ or $A_{i}$ (for some $i \in\{1,2\}$ ) is rational, $a_{i} \neq A_{i}$, and $b_{j}$ or $B_{j}$ (for some $j \in\{1,2\}$ ) is rational and $b_{j} \neq B_{j}$, then we would derive that the solution is constant, contrary to our assumption.

As an immediate consequence of Corollary 3, (A) and (B) we also obtain

Corollary 4. Suppose that (1) has a non-constant solution $f: X^{2} \rightarrow Y$ and assume that all coefficients $a_{i}, b_{i}, A_{i}, B_{i}$ for $i \in\{1,2\}$ are rational. Then

(a) if $A_{1}+A_{2} \neq 1$ then $\forall_{i \in\{1,2\}} a_{i}=A_{i}$;

(b) if $B_{1}+B_{2} \neq 1$ then $\forall_{i \in\{1,2\}} b_{i}=B_{i}$;

(c) $\forall_{i \in\{1,2\}} a_{i}=A_{i}$ or $\forall_{i \in\{1,2\}} b_{i}=B_{i}$.

In what follows we shall study the general solution of (1), so also these non-regular solutions of (1) or solutions of (1) with not necessarily rational coefficients. We start with two results from Kuczma's book [10], which we present here (adapted to the settings of the paper) for the convenience of the reader (cf., Lemmas 13.10.2, 13.10.3 therein).

Lemma 1. Given $a, b, A, B \in \mathbb{K}^{*}$, let $\xi: X \rightarrow Y$ be a non-zero additive function such that

$$
\xi(a x)=A \xi(x) \quad \text { and } \quad \xi(b x)=B \xi(x)
$$

for all $x \in X$. Assume that $r$ is a rational function in two variables with rational coefficients. If one of the expressions $r(a, b)$ and $r(A, B)$ makes sense, then the other makes sense, and

$$
\xi(r(a, b) x)=r(A, B) \xi(x), \quad x \in X .
$$

Lemma 2. Let $\Phi: \mathbb{Q}(a, b) \rightarrow \mathbb{Q}(A, B)$ be an isomorphism such that

$$
\Phi(a)=A, \quad \Phi(b)=B .
$$

Then $\left.\Phi\right|_{\mathbb{Q}}=i d$ and for every rational function $r \in \mathbb{Q}(x, y)$ such that $r(a, b)$, $r(A, B)$ make sense

$$
\Phi(r(a, b))=r(A, B) .
$$

Now we are ready to introduce our results concerning general solutions of (1).

Theorem 2. The condition $B_{1}+B_{2}=1$ is satisfied and

(H1) there exists an isomorphism $\Phi: \mathbb{Q}\left(a_{1}, a_{2}\right) \rightarrow \mathbb{Q}\left(A_{1}, A_{2}\right)$ such that $\Phi\left(a_{i}\right)=A_{i}, \quad i \in\{1,2\}$

if and only if there exists a non-trivial additive function $\varphi: X \rightarrow Y$ such that $f(x, y):=\varphi(x), x, y \in X$, is a solution of (1). 
Proof. In order to prove the sufficiency, let $H \subset X$ be a base of $X$ over $\mathbb{Q}\left(a_{1}, a_{2}\right)$ and let $\varphi_{0}: H \rightarrow Y$ be any function different from zero. For $x=$ $\sum_{i \in I} \alpha_{i} h_{i}$ with $\alpha_{i} \in \mathbb{Q}\left(a_{1}, a_{2}\right), h_{i} \in H(i \in I)$ we define

$$
\varphi(x):=\sum_{i \in I} \Phi\left(\alpha_{i}\right) \varphi_{0}\left(h_{i}\right) .
$$

One can check (cf., [10] or the proof of the forthcoming Proposition 1) that such a function is additive, uniquely determined and that $f$ defined in the theorem satisfies the first equation in (1). The assumption $B_{1}+B_{2}=1$ ensures that also the second equation in (1) is satisfied.

For necessity, assume that $\varphi \neq 0$ and define a function $\Phi: \mathbb{Q}\left(a_{1}, a_{2}\right) \rightarrow$ $\mathbb{Q}\left(A_{1}, A_{2}\right)$ by the formula

$$
\Phi\left(r\left(a_{1}, a_{2}\right)\right):=r\left(A_{1}, A_{2}\right)
$$

for any rational function $r$ with rational coefficients. The function is well defined. Indeed (cf., proof of [10, Theorem 13.10.4]), suppose that $r_{1}\left(a_{1}, a_{2}\right)=$ $r_{2}\left(a_{1}, a_{2}\right)$ for some $r_{1}, r_{2} \in \mathbb{Q}(x, y)$. There exists $x_{0} \in X$ such that $\varphi\left(x_{0}\right) \neq 0$. By Lemma 1 ,

$$
r_{1}\left(A_{1}, A_{2}\right) \varphi\left(x_{0}\right)=\varphi\left(r_{1}\left(a_{1}, a_{2}\right) x_{0}\right)=\varphi\left(r_{2}\left(a_{1}, a_{2}\right) x_{0}\right)=r_{2}\left(A_{1}, A_{2}\right) \varphi\left(x_{0}\right),
$$

whence $r_{1}\left(A_{1}, A_{2}\right)=r_{2}\left(A_{1}, A_{2}\right)$.

The function $\Phi$ is defined on the whole $\mathbb{Q}\left(a_{1}, a_{2}\right)$ and maps it onto $\mathbb{Q}\left(A_{1}, A_{2}\right)$ (see, e.g., [10, Corollary 4.8.1]). Also the rational functions $r_{x}(x, y)=\frac{x}{1}$ and $r_{y}(x, y)=\frac{y}{1}$ belong to $\mathbb{Q}(x, y)$, whence $\Phi\left(a_{1}\right)=\Phi\left(r_{x}\left(a_{1}, a_{2}\right)\right)=r_{x}\left(A_{1}, A_{2}\right)=$ $A_{1}$, and $\Phi\left(a_{2}\right)=\Phi\left(r_{y}\left(a_{1}, a_{2}\right)\right)=r_{y}\left(A_{1}, A_{2}\right)=A_{2} . \Phi$ is a homomorphism. Indeed, let $\alpha, \beta \in \mathbb{Q}\left(a_{1}, a_{2}\right)$. Then $\alpha=r_{1}\left(a_{1}, a_{2}\right), \beta=r_{2}\left(a_{1}, a_{2}\right)$ for some $r_{1}, r_{2} \in \mathbb{Q}(x, y)$ and

$$
\begin{aligned}
\Phi(\alpha+\beta) & =\Phi\left(\left(r_{1}+r_{2}\right)\left(a_{1}, a_{2}\right)\right)=\left(r_{1}+r_{2}\right)\left(A_{1}, A_{2}\right)=\Phi(\alpha)+\Phi(\beta), \\
\Phi(\alpha \beta) & =\Phi\left(\left(r_{1} r_{2}\right)\left(a_{1}, a_{2}\right)\right)=\left(r_{1} r_{2}\right)\left(A_{1}, A_{2}\right)=\Phi(\alpha) \Phi(\beta) .
\end{aligned}
$$

We will show that $\Phi$ is a monomorphism. Suppose that $\Phi(\alpha)=0$ for some $\alpha=r\left(a_{1}, a_{2}\right) \in \mathbb{Q}\left(a_{1}, a_{2}\right)$. Let $x_{0} \in X$ be such that $\varphi\left(x_{0}\right) \neq 0$. Suppose $\alpha \neq 0$. Then on account of Lemma 1 ,

$$
0=r\left(A_{1}, A_{2}\right) \varphi\left(\frac{x_{0}}{r\left(a_{1}, a_{2}\right)}\right)=\varphi\left(x_{0}\right) \neq 0 .
$$

This contradiction shows that $\Phi(\alpha)=0$ implies $\alpha=0$, that is, $\Phi$ is a monomorphism. Since it is also an epimorphism (as pointed out above), $\Phi$ is an isomorphism.

Analogously, we get the following.

Theorem 3. The condition $A_{1}+A_{2}=1$ holds and

$(\mathrm{H} 2)$ there exists an isomorphism $\Psi: \mathbb{Q}\left(b_{1}, b_{2}\right) \rightarrow \mathbb{Q}\left(B_{1}, B_{2}\right)$ such that $\Psi\left(b_{i}\right)=$ $B_{i}, \quad i \in\{1,2\}$ 
if and only if there exists an non-trivial additive function $\psi: X \rightarrow Y$ such that $f(x, y):=\psi(y), x, y \in X$, is a solution of $(1)$.

In the next results we are interested in the biadditive solutions of (1).

Proposition 1. Suppose that (H1) and (H2) hold. Let $H, L \subset X$ be bases of $X$ over $\mathbb{Q}\left(a_{1}, a_{2}\right)$ and $\mathbb{Q}\left(b_{1}, b_{2}\right)$, respectively. For every function $g_{0}: H \times L \rightarrow Y$ such that there exists a unique biadditive function $g: X^{2} \rightarrow Y$ satisfying (1) and such that $\left.g\right|_{H \times L}=g_{0}$.

Proof. Take $x, y \in X$. Then

$$
x=\sum_{i=1}^{n} \alpha_{i} h_{i}, \quad y=\sum_{j=1}^{m} \beta_{j} l_{j},
$$

where $\alpha_{i} \in \mathbb{Q}\left(a_{1}, a_{2}\right), \beta_{j} \in \mathbb{Q}\left(b_{1}, b_{2}\right)$ and $h_{i} \in H, l_{j} \in L$ for $i \in\{1, \ldots, n\}$, $j \in\{1, \ldots, m\}$. Define $g: X^{2} \rightarrow Y$ by the formula

$$
g(x, y):=\sum_{i=1}^{n} \sum_{j=1}^{m} \Phi\left(\alpha_{i}\right) \Psi\left(\beta_{j}\right) g_{0}\left(h_{i}, l_{j}\right) .
$$

We check that $g$ defined by (13) satisfies (1). Take $x_{1}=\sum_{i=1}^{n} \alpha_{i}^{\prime} h_{i}, x_{2}=$ $\sum_{i=1}^{n} \alpha_{i}^{\prime \prime} h_{i}, y=\sum_{j=1}^{m} \beta_{j} l_{j}$ from $X$. Then

$$
\begin{aligned}
g\left(a_{1} x_{1}+a_{2} x_{2}, y\right)= & g\left(\sum_{i=1}^{n}\left(\alpha_{i}^{\prime} a_{1}+\alpha_{i}^{\prime \prime} a_{2}\right) h_{i}, \sum_{j=1}^{m} \beta_{j} l_{j}\right) \\
= & \sum_{i=1}^{n} \sum_{j=1}^{m} \Phi\left(\alpha_{i}^{\prime} a_{1}+\alpha_{i}^{\prime \prime} a_{2}\right) \Psi\left(\beta_{j}\right) g_{0}\left(h_{i}, l_{j}\right) \\
= & \sum_{i=1}^{n} \sum_{j=1}^{m}\left(A_{1} \Phi\left(\alpha_{i}^{\prime}\right)+A_{2} \Phi\left(\alpha_{i}^{\prime \prime}\right)\right) \Psi\left(\beta_{j}\right) g_{0}\left(h_{i}, l_{j}\right) \\
= & A_{1} \sum_{i=1}^{n} \sum_{j=1}^{m} \Phi\left(\alpha_{i}^{\prime}\right) \Psi\left(\beta_{j}\right) g_{0}\left(h_{i}, l_{j}\right) \\
& +A_{2} \sum_{i=1}^{n} \sum_{j=1}^{m} \Phi\left(\alpha_{i}^{\prime \prime}\right) \Psi\left(\beta_{j}\right) g_{0}\left(h_{i}, l_{j}\right) \\
= & A_{1} g\left(\sum_{i=1}^{n} \alpha_{i}^{\prime} h_{i}, \sum_{j=1}^{m} \beta_{j} l_{j}\right)+A_{2} g\left(\sum_{i=1}^{n} \alpha_{i}^{\prime \prime} h_{i}, \sum_{j=1}^{m} \beta_{j} l_{j}\right) \\
= & A_{1} g\left(x_{1}, y\right)+A_{2} g\left(x_{2}, y\right),
\end{aligned}
$$

and analogously, we get

$$
g\left(x, b_{1} y_{1}+b_{2} y_{2}\right)=B_{1} g\left(x, y_{1}\right)+B_{2} g\left(x, y_{2}\right) .
$$


Moreover, (if $x=h_{i}$ then $\alpha_{i}=1$ and $\alpha_{k}=0$ for all $k \neq i$; if $y=l_{j}$ then $\beta_{j}=1$ and $\beta_{k}=0$ for all $k \neq j$; if $x=0$ or $y=0$ then $\alpha_{i}=0$ for all $i$ or $\beta_{j}=0$ for all $j$; cf., Lemma 2)

$$
g\left(h_{i}, l_{j}\right)=g_{0}\left(h_{i}, l_{j}\right) .
$$

In order to prove the uniqueness, assume that $g$ satisfies (1) and $\left.g\right|_{H \times L}=g_{0}$. For every $i \in\{1, \ldots, n\}$ there exist a rational function $r_{i} \in \mathbb{Q}(x, y)$ such that $\alpha_{i}=r_{i}\left(a_{1}, a_{2}\right)$. Similarly, for every $j \in\{1, \ldots, m\}$ there exists a rational function $s_{j} \in \mathbb{Q}(x, y)$ such that $\beta_{j}=s_{j}\left(b_{1}, b_{2}\right)$.

By Lemmas 1 and 2,

$$
\begin{aligned}
g(x, y) & =g\left(\sum_{i=1}^{n} \alpha_{i} h_{i}, \sum_{j=1}^{m} \beta_{j} l_{j}\right)=\sum_{i=1}^{n} \sum_{j=1}^{m} g\left(\alpha_{i} h_{i}, \beta_{j} l_{j}\right) \\
& =\sum_{i=1}^{n} \sum_{j=1}^{m} g\left(r_{i}\left(a_{1}, a_{2}\right) h_{i}, s_{j}\left(b_{1}, b_{2}\right) l_{j}\right) \\
& =\sum_{i=1}^{n} \sum_{j=1}^{m} r_{i}\left(A_{1}, A_{2}\right) s_{j}\left(B_{1}, B_{2}\right) g\left(h_{i}, l_{j}\right) \\
& =\sum_{i=1}^{n} \sum_{j=1}^{m} \Phi\left(r_{i}\left(a_{1}, a_{2}\right)\right) \Psi\left(s_{j}\left(b_{1}, b_{2}\right)\right) g\left(h_{i}, l_{j}\right) \\
& =\sum_{i=1}^{n} \sum_{j=1}^{m} \Phi\left(\alpha_{i}\right) \Psi\left(\beta_{j}\right) g_{0}\left(h_{i}, l_{j}\right) .
\end{aligned}
$$

Consequently, $g$ must be given by the formula (13), which proves the uniqueness and finishes the proof.

Theorem 4. Hypotheses (H1) and (H2) hold if and only if there exists a nonzero biadditive function $\mathrm{g}: X^{2} \rightarrow Y$ satisfying (1).

Proof. The sufficiency follows from Proposition 1, and the proof of necessity is done similarly to that in Theorem 2 .

Corollary 5. Suppose (1) has a non-constant solution $f: X^{2} \rightarrow Y$. Then (H1) or (H2) holds true.

Proof. It is enough to observe that system (1) has a non-constant solution $f: X^{2} \rightarrow Y$, that is, using the form (4) of $f$,

$g \neq 0 \quad$ or $\quad \varphi \neq 0 \quad$ or $\quad \psi \neq 0$.

As an immediate consequence of Theorems 1, 2, 3, 4 and Corollary 5 we are able to state the following. 
Corollary 6. System (1) has a non-constant solution $f: X^{2} \rightarrow Y$ if and only if at least one of the following conditions holds

(i) (H1) and (H2);

(ii) (H1) and $B_{1}+B_{2}=1$;

(iii) (H2) and $A_{1}+A_{2}=1$.

\section{Solutions of $(3)$}

In what follows we shall study (3). We start with the following result (see also [9] in which a function with values in an arbitrary field of characteristic different from two is considered).

Theorem 5. If $f: X^{2} \rightarrow Y$ satisfies (3), then there exist a biadditive function $g: X^{2} \rightarrow Y$, additive functions $\varphi, \psi: X \rightarrow Y$ and a constant $\delta \in Y$ such that $f$ has the form (4) and for all $x, y \in X$,

$$
\begin{aligned}
& g\left(a_{1} x, b_{1} y\right)=C_{1} g(x, y), \\
& g\left(a_{1} x, b_{2} y\right)=C_{2} g(x, y), \\
& g\left(a_{2} x, b_{1} y\right)=C_{3} g(x, y), \\
& g\left(a_{2} x, b_{2} y\right)=C_{4} g(x, y), \\
& \varphi\left(a_{1} x\right)=\left(C_{1}+C_{2}\right) \varphi(x), \\
& \varphi\left(a_{2} x\right)=\left(C_{3}+C_{4}\right) \varphi(x), \\
& \psi\left(b_{1} y\right)=\left(C_{1}+C_{3}\right) \psi(y), \\
& \psi\left(b_{2} y\right)=\left(C_{2}+C_{4}\right) \psi(y),
\end{aligned}
$$

and

$$
\delta\left(C_{1}+C_{2}+C_{3}+C_{4}-1\right)=0 .
$$

Conversely, each function $f$ of the form (4) with $g$ biadditive, $\varphi, \psi$ additive, and such that conditions (14), (15), (16), (17) are satisfied, is a solution of (3).

Proof. Assume $f: X^{2} \rightarrow Y$ satisfies (3). From (3), for all $x_{1}, x_{2}, y_{1}, y_{2} \in X$ we have

$$
\begin{aligned}
& f\left(a_{1} x_{1}, b_{1} y_{1}\right)=C_{1} f\left(x_{1}, y_{1}\right)+C_{2} f\left(x_{1}, 0\right)+C_{3} f\left(0, y_{1}\right)+C_{4} f(0,0), \\
& f\left(a_{1} x_{1}, b_{2} y_{2}\right)=C_{1} f\left(x_{1}, 0\right)+C_{2} f\left(x_{1}, y_{2}\right)+C_{3} f(0,0)+C_{4} f\left(0, y_{2}\right), \\
& f\left(a_{2} x_{2}, b_{1} y_{1}\right)=C_{1} f\left(0, y_{1}\right)+C_{2} f(0,0)+C_{3} f\left(x_{2}, y_{1}\right)+C_{4} f\left(x_{2}, 0\right), \\
& f\left(a_{2} x_{2}, b_{2} y_{2}\right)=C_{1} f(0,0)+C_{2} f\left(0, y_{2}\right)+C_{3} f\left(x_{2}, 0\right)+C_{4} f\left(x_{2}, y_{2}\right) .
\end{aligned}
$$

Moreover, for all $x_{1}, x_{2}, y_{1}, y_{2} \in X$,

$$
\begin{aligned}
& f\left(a_{1} x_{1}, 0\right)=\left(C_{1}+C_{2}\right) f\left(x_{1}, 0\right)+\left(C_{3}+C_{4}\right) f(0,0), \\
& f\left(a_{2} x_{2}, 0\right)=\left(C_{3}+C_{4}\right) f\left(x_{2}, 0\right)+\left(C_{1}+C_{2}\right) f(0,0), \\
& f\left(0, b_{1} y_{1}\right)=\left(C_{1}+C_{3}\right) f\left(0, y_{1}\right)+\left(C_{2}+C_{4}\right) f(0,0), \\
& f\left(0, b_{2} y_{2}\right)=\left(C_{2}+C_{4}\right) f\left(0, y_{2}\right)+\left(C_{1}+C_{3}\right) f(0,0) .
\end{aligned}
$$


From (3), (18) and (19) we obtain

$$
\begin{aligned}
f\left(a_{1} x_{1}+a_{2} x_{2}, b_{1} y_{1}+b_{2} y_{2}\right)= & f\left(a_{1} x_{1}, b_{1} y_{1}\right)+f\left(a_{1} x_{1}, b_{2} y_{2}\right) \\
& +f\left(a_{2} x_{2}, b_{1} y_{1}\right)+f\left(a_{2} x_{2}, b_{2} y_{2}\right) \\
& -f\left(a_{1} x_{1}, 0\right)-f\left(a_{2} x_{2}, 0\right) \\
& -f\left(0, b_{1} y_{1}\right)-f\left(0, b_{2} y_{2}\right)+f(0,0),
\end{aligned}
$$

and since $a_{1} a_{2} b_{1} b_{2} \neq 0$, we have

$$
\begin{aligned}
f\left(x_{1}+x_{2}, y_{1}+y_{2}\right)= & f\left(x_{1}, y_{1}\right)+f\left(x_{1}, y_{2}\right)+f\left(x_{2}, y_{1}\right)+f\left(x_{2}, y_{2}\right) \\
& -f\left(x_{1}, 0\right)-f\left(x_{2}, 0\right)-f\left(0, y_{1}\right)-f\left(0, y_{2}\right) \\
& +f(0,0),
\end{aligned}
$$

for all $x_{1}, x_{2}, y_{1}, y_{2} \in X$.

Immediately from (20) we get

$$
f\left(0, y_{1}+y_{2}\right)=f\left(0, y_{1}\right)+f\left(0, y_{2}\right)-f(0,0), \quad y_{1}, y_{2} \in X,
$$

and

$$
f\left(x_{1}+x_{2}, 0\right)=f\left(x_{1}, 0\right)+f\left(x_{2}, 0\right)-f(0,0), \quad x_{1}, x_{2} \in X,
$$

that is, $\psi(y):=f(0, y)-f(0,0)$ for $y \in X$ and $\varphi(x):=f(x, 0)-f(0,0)$ for $x \in X$ are additive. It is easy to check that the function $g(x, y):=f(x, y)-$ $f(0, y)-f(x, 0)+f(0,0)$ for all $x, y \in X$ is biadditive and

$f(x, y)=g(x, y)+(f(x, 0)-f(0,0))+(f(0, y)-f(0,0))+f(0,0), x, y \in X$,

which means that $f$ has the form (4) with $\delta=f(0,0)$.

Substituting this form into (3), with $x_{1}=x_{2}=y_{1}=y_{2}=0$ we get $\delta=\left(C_{1}+C_{2}+C_{3}+C_{4}\right) \delta$, that is, we have (17).

Now, it is enough to apply (3) with $f(x, y)=g(x, y)+\varphi(x)+\psi(y)$ for $x, y \in X$. Suitable fixing of variables $x_{1}, x_{2}, y_{1}, y_{2}$ immediately gives (14), (15), (16).

The converse implication we obtain by a direct computation.

Similarly to the previous section, we start with studying solutions of (3) in some special cases. For this purpose we introduce the following conditions:

$$
\begin{gathered}
a_{1} b_{1}=C_{1} \wedge a_{1} b_{2}=C_{2} \wedge a_{2} b_{1}=C_{3} \wedge a_{2} b_{2}=C_{4}, \\
C_{1}+C_{2}=a_{1} \wedge C_{3}+C_{4}=a_{2}, \\
C_{1}+C_{3}=b_{1} \wedge C_{2}+C_{4}=b_{2}, \\
C_{1}+C_{2}+C_{3}+C_{4}=1 .
\end{gathered}
$$


Corollary 7. Let $f: X^{2} \rightarrow Y$ satisfy (3) and let coefficients $a_{i}, b_{i}, i \in\{1,2\}$, be rational. Then there exist a biadditive function $g: X^{2} \rightarrow Y$, additive functions $\varphi, \psi: X \rightarrow Y$ and a constant $\delta \in Y$ such that $f$ has the form (4) and

$$
\begin{aligned}
& g=0, \text { whenever } \sim(c \alpha) \\
& \varphi=0, \text { whenever } \sim(c \beta) ; \\
& \psi=0, \text { whenever } \sim(c \gamma) ; \\
& \delta=0, \text { whenever } \sim(c \delta) .
\end{aligned}
$$

Remark 3. Notice that

- $[(c \alpha) \wedge(c \beta) \wedge \sim(c \gamma)] \Rightarrow \sim(c \delta)$ (because then $b_{1}+b_{2}=1$ and $C_{1}+C_{2}+C_{3}+C_{4}=a_{1}+a_{2} \neq 1$ ),

- $[(c \alpha) \wedge \sim(c \beta) \wedge(c \gamma)] \Rightarrow \sim(c \delta)$

(because then $a_{1}+a_{2}=1$ and $C_{1}+C_{2}+C_{3}+C_{4}=b_{1}+b_{2} \neq 1$ ).

Moreover, we have $[(c \alpha) \wedge(c \beta) \wedge(c \gamma)] \Rightarrow(c \delta)$.

As a consequence of Theorem 5 we also have the following.

Corollary 8. Let $f: \mathbb{R}^{2} \rightarrow \mathbb{R}$ satisfy any regularity condition that forces a biadditive function to be continuous. The general regular solution of (3) is of the form

$$
f(x, y)=\alpha x y+\beta x+\gamma y+\delta, \quad x, y \in \mathbb{R},
$$

where $\alpha, \beta, \gamma, \delta \in \mathbb{R}$ are arbitrary and such that

$$
\begin{aligned}
& \alpha=0, \text { whenever } \sim(c \alpha) \\
& \beta=0, \text { whenever } \sim(c \beta) ; \\
& \gamma=0, \text { whenever } \sim(c \gamma) ; \\
& \delta=0, \text { whenever } \sim(c \delta) .
\end{aligned}
$$

Remark 4. By Corollaries 7 and 8, in the two special cases it follows that

- there exists a non-zero biadditive $g$ which is a solution of (3) if and only if $(c \alpha)$ holds;

- there exists a non-zero additive function $\varphi$ such that $f(x, y):=\varphi(x)$ is a solution of (3) if and only if $(c \beta)$ holds;

- there exists a non-zero additive function $\psi$ such that $f(x, y):=\psi(y)$ is a solution of (3) if and only if $(c \gamma)$ holds;

- there exists $\delta \neq 0$ such that function $f(x, y) \equiv \delta$ is a solution of $(3)$ if and only if $(c \delta)$ holds.

Before presenting the general solution of (3), we make some remarks and observations.

As an immediate consequence of Theorem 5 we obtain 
Corollary 9. A function $f: X^{2} \rightarrow Y$ of the form (4) is a solution of (3) if and only if $g$ is biadditive and satisfies (14), $\varphi, \psi$ are additive functions satisfying the general linear equations

$$
\begin{aligned}
& \varphi\left(a_{1} x_{1}+a_{2} x_{2}\right)=\left(C_{1}+C_{2}\right) \varphi\left(x_{1}\right)+\left(C_{3}+C_{4}\right) \varphi\left(x_{2}\right), \quad x_{1}, x_{2} \in X \\
& \psi\left(b_{1} y_{1}+b_{2} y_{2}\right)=\left(C_{1}+C_{3}\right) \psi\left(y_{1}\right)+\left(C_{2}+C_{4}\right) \psi\left(y_{2}\right), \quad y_{1}, y_{2} \in X
\end{aligned}
$$

respectively, and $\delta$ satisfies (17).

Concerning non-constant solutions of (3), the following facts are of interest. Remark 5. A non-constant function $f$ is a solution of (3) if and only if $g \neq 0$ or $\varphi \neq 0$ or $\psi \neq 0$.

Remark 6. If $g$ is a non-zero function satisfying (14) then $C_{1} C_{4}=C_{2} C_{3}$. Indeed,

$$
C_{1} C_{4} g(x, y)=g\left(a_{1} a_{2} x, b_{1} b_{2} y\right)=C_{2} C_{3} g(x, y)
$$

moreover

$$
\begin{aligned}
& C_{1} g\left(x, b_{2} y\right)=g\left(a_{1} x, b_{1} b_{2} y\right)=C_{2} g\left(x, b_{1} y\right), \\
& C_{3} g\left(x, b_{2} y\right)=g\left(a_{2} x, b_{1} b_{2} y\right)=C_{4} g\left(x, b_{1} y\right), \\
& C_{1} g\left(a_{2} x, y\right)=g\left(a_{1} a_{2} x, b_{1} y\right)=C_{3} g\left(a_{1} x, y\right), \\
& C_{2} g\left(a_{2} x, y\right)=g\left(a_{1} a_{2} x, b_{2} y\right)=C_{4} g\left(a_{1} x, y\right) .
\end{aligned}
$$

In what follows we give an example of a general bilinear equation where not all coefficients are rational which has a non-constant solution and later on we proceed with general considerations concerning non-constant solutions of (3).

Example 2. Consider the following equation

$$
\begin{aligned}
f\left(2 x_{1}+\sqrt{2} x_{2}, \sqrt{3} y_{1}+\sqrt{5} y_{2}\right)= & f\left(x_{1}, y_{1}\right)+f\left(x_{1}, y_{2}\right)+\pi f\left(x_{2}, y_{1}\right) \\
& +(\sqrt{2}-\pi) f\left(x_{2}, y_{2}\right)
\end{aligned}
$$

for all $x_{1}, x_{2}, y_{1}, y_{2} \in X$. One can easily check that

$$
f(x, y)=\varphi(x), \quad x, y \in X,
$$

where $\varphi: X \rightarrow Y$ is an arbitrary additive function satisfying

$$
\varphi(\sqrt{2} x)=\sqrt{2} \varphi(x), \quad x \in X
$$

is a solution to $(3)$.

In the sequel, we describe results concerning each of the functions $\varphi, \psi$ and $g$ appearing in the form (4) separately.

Theorem 6. The function $f: X^{2} \rightarrow Y$ given by (24) with some non-zero additive function $\varphi: X \rightarrow Y$ satisfies (3) if and only if 
(H3) there exists an isomorphism $\Gamma: \mathbb{Q}\left(a_{1}, a_{2}\right) \rightarrow \mathbb{Q}\left(C_{1}+C_{2}, C_{3}+C_{4}\right)$ such that $\Gamma\left(a_{1}\right)=C_{1}+C_{2}$ and $\Gamma\left(a_{2}\right)=C_{3}+C_{4}$.

Proof. By Corollary 9, function $f$ given by (24) satisfies (3) if and only if $\varphi$ satisfies the general linear equation (22). This fact is therefore equivalent (see, e.g., Kuczma [10, Theorem 13.10.4]) to (H3).

Analogously, with the use of (23), we obtain

Theorem 7. The function $f: X^{2} \rightarrow Y$ given by $f(x, y):=\psi(y)$ for all $x, y \in X$ with some non-zero additive function $\psi: X \rightarrow Y$ satisfies (3) if and only if

(H4) there exists an isomorphism $\Lambda: \mathbb{Q}\left(b_{1}, b_{2}\right) \rightarrow \mathbb{Q}\left(C_{1}+C_{3}, C_{2}+C_{4}\right)$ such that $\Lambda\left(b_{1}\right)=C_{1}+C_{3}$ and $\Lambda\left(b_{2}\right)=C_{2}+C_{4}$.

It is obvious that if both $\varphi$ and $\psi$ are not constant, then $\Gamma\left(a_{1}\right)+\Gamma\left(a_{2}\right)=$ $\Lambda\left(b_{1}\right)+\Lambda\left(b_{2}\right)=C_{1}+C_{2}+C_{3}+C_{4}$.

In what follows we study biadditive solutions of (3).

Proposition 2. Assume $a_{i}, b_{i} \in \mathbb{K}^{*}, C_{j} \in \mathbb{K}, i \in\{1,2\}, j \in\{1,2,3,4\}$. If there exist $A_{i}, B_{i} \in \mathbb{K}, i \in\{1,2\}$, such that

(H5) $C_{1}=A_{1} B_{1}, C_{2}=A_{1} B_{2}, C_{3}=A_{2} B_{1}, C_{4}=A_{2} B_{2}$, and both hypotheses (H1) and (H2) hold

then for every function $g_{0}: H \times L \rightarrow Y$, there exists a unique biadditive function $g: X^{2} \rightarrow Y$ satisfying (3) and such that $\left.g\right|_{H \times L}=g_{0}$, where $H, L \subset X$ are bases of $X$ over $\mathbb{Q}\left(a_{1}, a_{2}\right)$ and $\mathbb{Q}\left(b_{1}, b_{2}\right)$, respectively.

Proof. We notice that the assumptions of Theorem 4 are satisfied, so for every function $g_{0}: H \times L \rightarrow Y$, there exists a unique biadditive function $g: X^{2} \rightarrow$ $Y$ satisfying (1) and such that $\left.g\right|_{H \times L}=g_{0}$. As a consequence, since $C_{1}=$ $A_{1} B_{1}, C_{2}=A_{1} B_{2}, C_{3}=A_{2} B_{1}, C_{4}=A_{2} B_{2}, g$ satisfies also (3).

As a consequence of the above we can state

Theorem 8. Assume $a_{i}, b_{i} \in \mathbb{K}^{*}, C_{j} \in \mathbb{K}, i \in\{1,2\}, j \in\{1,2,3,4\}$. If there exist $A_{i}, B_{i} \in \mathbb{K}, i \in\{1,2\}$, such that (H5) holds, then there exists a non-zero biadditive function $g: X^{2} \rightarrow Y$ satisfying (3) and, moreover, (5).

Proof. By the construction of a solution in Proposition 2, $g$ satisfies (1). Hence, using Theorem 1, it satisfies (5).

Remark 7. Since $a_{i}, b_{i} \in \mathbb{K}^{*}$, so do their isomorphic images, whenever (H5) is satisfied. Therefore, also $C_{j} \in \mathbb{K}^{*}$. Moreover, if at least one of $a_{1}, a_{2}, b_{1}, b_{2}$ is rational, its image being the same number is uniquely determined, and the conditions $C_{1}=A_{1} B_{1}, C_{2}=A_{1} B_{2}, C_{3}=A_{2} B_{1}, C_{4}=A_{2} B_{2}$ uniquely determine the other numbers from $A_{1}, A_{2}, B_{1}, B_{2}$. 
Remark 8. For every $C_{1}, C_{2}, C_{3}, C_{4} \in \mathbb{K}^{*}$ such that $C_{1} C_{4}=C_{2} C_{3}$ there exist $\bar{A}_{1}, \bar{A}_{2}, \bar{B}_{1}, \bar{B}_{2}$ from $\mathbb{K}^{*}$ such that

$$
C_{1}=\bar{A}_{1} \bar{B}_{1}, C_{2}=\bar{A}_{1} \bar{B}_{2}, C_{3}=\bar{A}_{2} \bar{B}_{1}, C_{4}=\bar{A}_{2} \bar{B}_{2} .
$$

Indeed, it is enough to choose one of the elements $\bar{A}_{1}, \bar{A}_{2}, \bar{B}_{1}, \bar{B}_{2}$ and the others are already clearly defined. For example, if we choose $\bar{A}_{1}$ then

$$
\bar{B}_{1}=\frac{C_{1}}{\bar{A}_{1}}, \bar{B}_{2}=\frac{C_{2}}{\bar{A}_{1}} \text { and } \bar{A}_{2}=\frac{C_{3}}{\bar{B}_{1}} \text {. }
$$

We will come back now to Example 2.

Remark 9. Based on the above results one can see that in fact function $f$ of the form (24) is the general solution of the equation

$$
\begin{aligned}
f\left(2 x_{1}+\sqrt{2} x_{2}, \sqrt{3} y_{1}+\sqrt{5} y_{2}\right)= & f\left(x_{1}, y_{1}\right)+f\left(x_{1}, y_{2}\right)+\pi f\left(x_{2}, y_{1}\right) \\
& +(\sqrt{2}-\pi) f\left(x_{2}, y_{2}\right)
\end{aligned}
$$

for all $x_{1}, x_{2}, y_{1}, y_{2} \in X$, where $\varphi: X \rightarrow Y$ is an arbitrary additive function satisfying condition (25). Indeed, by Theorem 5 the solutions $f$ are of the form (4) with (14), (15), (16) and (17) satisfied. Since $C_{1} C_{4}=\sqrt{2}-\pi \neq \pi=C_{2} C_{3}$, on account of Remark 6 , the biadditive function $g$ equals zero. The existence of an additive function $\varphi: X \rightarrow Y$ is guaranteed by Theorem 6. Further, none of the elements $\sqrt{3}, \sqrt{5}$ is conjugated to any of $\pi+1,1+\sqrt{2}-\pi$, so no isomorphism $\Psi: \mathbb{Q}(\sqrt{3}, \sqrt{5}) \rightarrow \mathbb{Q}(\pi+1,1+\sqrt{2}-\pi)$ exists [10, Theorem 4.12.2], and by Theorem $7, \psi=0$. Finally, since $C_{1}+C_{2}+C_{3}+C_{4} \neq 1$, we have $\delta=0$.

From Remark 5 and Theorems 6, 7, 8 we get the following.

Theorem 9. Assume $f: X^{2} \rightarrow Y$. If at least one of the hypotheses (H3), (H4) holds or there exist $A_{1}, A_{2}, B_{1}, B_{2} \in \mathbb{K}^{*}$ such that (H5) holds then there exists a non-constant solution of (3).

Theorem 8, and so Theorem 9, give a sufficient condition for the existence of a non-constant solution of (3). Some approach for obtaining the necessary conditions in the case $Y$ is a field is given in [9, Section 6]. Therefore it is worth finishing this section with formulating a problem.

Problem 1. Find the general (biadditive) solution $f: X^{2} \rightarrow Y$ of (3).

\section{Equivalence of (1) and (3)}

Comparing the form of solutions of (1) and (3), we come back to the question about conditions which have to be satisfied for (1) and (3) to be equivalent. First we answer this question in two special cases: for equations with rational coefficients and when we are looking for regular solutions $f: \mathbb{R}^{2} \rightarrow \mathbb{R}$. 
On account of Remarks 2 and 4 we are able to prove the following result which completely describes the equivalence between (1) and (3) in these cases.

Theorem 10. Let $f: X^{2} \rightarrow Y$ and $a_{1}, a_{2}, b_{1}, b_{2} \in \mathbb{Q}^{*}$ or let $f: \mathbb{R}^{2} \rightarrow \mathbb{R}$ be $a$ regular solution of (1) or (3). Then (1) and (3) are equivalent (have the same set of solutions) if and only if one of the following conditions hold:

$$
\begin{aligned}
1^{\circ} & (\alpha) \wedge(c \alpha) \wedge\left(\left(a_{1}+a_{2}=b_{1}+b_{2}=1\right) \vee\left(a_{1}+a_{2}\right)\left(b_{1}+b_{2}\right) \neq 1\right) ; \\
2^{\circ} & \forall_{i \in\{1,2\}} a_{i}=A_{i} \wedge \exists_{i \in\{1,2\}} b_{i} \neq B_{i} \wedge B_{1}+B_{2}=1 \wedge \sim(c \alpha) \wedge(c \beta) \wedge \\
& \sim(c \gamma) ; \\
3^{\circ} & \exists_{i \in\{1,2\}} a_{i} \neq A_{i} \wedge \forall_{i \in\{1,2\}} b_{i}=B_{i} \wedge A_{1}+A_{2}=1 \wedge \sim(c \alpha) \wedge \\
& \sim(c \beta) \wedge(c \gamma) ; \\
4^{\circ} & \sim((\alpha) \vee(c \alpha) \vee(\beta) \vee(c \beta) \vee(\gamma) \vee(c \gamma)) \\
& \wedge\left(C_{1}+C_{2}+C_{3}+C_{4}=A_{1}+A_{2}=B_{1}+B_{2}=1\right. \\
& \left.\vee\left(C_{1}+C_{2}+C_{3}+C_{4} \neq 1 \wedge\left(A_{1}+A_{2}\right)\left(B_{1}+B_{2}\right) \neq 1\right)\right) .
\end{aligned}
$$

Proof. Assume that (1) and (3) are equivalent.

I. We start with the case when there exists a non-zero biadditive function $g$ which is a solution of both (1) and (3), that is, by Remarks 2 and 4, $(\alpha)$ and $(c \alpha)$ are satisfied.

Since under the above assumptions we have

$$
\left(C_{1}+C_{2}=a_{1} \wedge C_{3}+C_{4}=a_{2}\right) \Leftrightarrow B_{1}+B_{2}=1
$$

and

$$
\left(C_{1}+C_{3}=b_{1} \wedge C_{2}+C_{4}=b_{2}\right) \Leftrightarrow A_{1}+A_{2}=1,
$$

it is crucial that $(\delta)$ and $(c \delta)$ hold simultaneously, and this we have if either $a_{1}+a_{2}=b_{1}+b_{2}=1$, or $\left(a_{1}+a_{2}\right)\left(b_{1}+b_{2}\right) \neq 1$. Consequently, we have obtained $1^{\circ}$.

II. Assume that only the zero biadditive function $g$ satisfies (1) and (3).

Observe first that it is impossible that the solution in such a case consists of both a non-zero $\varphi$ and a non-zero $\psi$. Indeed, in such a case $(\alpha)$ would hold, contrary to the assumption.

Assume that there exists a non-zero $\varphi$ and only $\psi=0$ forms the solution. Then we have $\sim(\alpha), \sim(\mathrm{c} \alpha),(\beta),(\mathrm{c} \beta), \sim(\gamma)$ and $\sim(\mathrm{c} \gamma)$. By $\sim(\alpha)$ and $(\beta)$ we have $\forall_{i \in\{1,2\}} a_{i}=A_{i}, \exists_{i \in\{1,2\}} b_{i} \neq B_{i}$ and $B_{1}+B_{2}=1$. By (c $\beta$ ) it follows that $C_{1}+C_{2}+C_{3}+C_{4}=a_{1}+a_{2}=A_{1}+A_{2}$. This means that $(\delta)$ and $(\mathrm{c} \delta)$ are equivalent, and we have $2^{\circ}$.

Assume that there exists a non-zero $\psi$ and only the zero functions $g$ and $\varphi$ are parts of the solution of (1) and (3). Then we have $\sim(\alpha), \sim(c \alpha), \sim(\beta)$, $\sim(\mathrm{c} \beta),(\gamma)$ and $(\mathrm{c} \gamma)$, and we proceed analogously as above, obtaining $3^{\circ}$.

Assume finally, that there are only constant solutions of (1) and (3), that is, $\sim(\alpha), \sim(c \alpha), \sim(\beta), \sim(\mathrm{c} \beta), \sim(\gamma)$ and $\sim(\mathrm{c} \gamma)$ hold. That means that either any non-zero constant or only $f(x, y) \equiv 0$ is a solution both to (1) and (3). Therefore, either $C_{1}+C_{2}+C_{3}+C_{4}=A_{1}+A_{2}=B_{1}+B_{2}=1$ or $\left(C_{1}+C_{2}+\right.$ 
$C_{3}+C_{4} \neq 1$ and $\left(A_{1}+A_{2} \neq 1\right.$ or $\left.\left.B_{1}+B_{2} \neq 1\right)\right)$. The above conditions gives $4^{\circ}$.

For the converse, assume now that $1^{\circ}$ holds. It means that function $f(x, y)=$ $g(x, y)+\varphi(x)+\psi(y)+\delta$ with any biadditive $g$ satisfies both (1) and (3). If also $a_{1}+a_{2}=b_{1}+b_{2}=1$, then $(\beta),(\gamma)$ and $(\delta)$. And by $(\alpha)$, also $(\mathrm{c} \beta),(\mathrm{c} \gamma)$ and $(\mathrm{c} \delta)$. All further computations are direct on account of Remarks 2 and 4.

The next result solves the problem of alienation answering the question when, given (3), there exist $A_{1}, A_{2}, B_{1}, B_{2} \in \mathbb{K}$ such that $f$ satisfying (3) is also a solution of system (1), so in other words, when (3) "splits" into system (1) of two equations? It follows directly from Theorem 10.

Theorem 11. Let $f: X^{2} \rightarrow Y$ be a solution of $(3)$ with $a_{1}, a_{2}, b_{1}, b_{2} \in \mathbb{Q}^{*}$ or let $f: \mathbb{R}^{2} \rightarrow \mathbb{R}$ be a regular solution of (3). Define $A_{i}, B_{i}, i \in\{1,2\}$ :

$1^{\circ}$ if $(c \alpha)$ and $\left(a_{1}+a_{2}=b_{1}+b_{2}=1\right) \vee\left(a_{1}+a_{2}\right)\left(b_{1}+b_{2}\right) \neq 1$, then take $A_{i}:=a_{i}$ and $B_{i}:=b_{i}, i \in\{1,2\}$;

$2^{\circ}$ if $\sim(c \alpha),(c \beta)$ and $\sim(c \gamma)$, then take $A_{i}:=a_{i}$ and $B_{i}, i \in\{1,2\}$, such that $B_{1}+B_{2}=1$ and $B_{i} \neq b_{i}$ for some $i \in\{1,2\}$;

$3^{\circ}$ if $\sim(c \alpha), \sim(c \beta)$ and $(c \gamma)$, then take $B_{i}:=b_{i}$ and $A_{i}, i \in\{1,2\}$, such that $A_{1}+A_{2}=1$ and $A_{i} \neq a_{i}$ for some $i \in\{1,2\}$;

$4^{\circ}$ if $\sim(c \alpha), \sim(c \beta)$ and $\sim(c \gamma)$, then take arbitrary $A_{i}, B_{i}, i \in\{1,2\}$, such that $A_{i} \neq a_{i}$ for some $i \in\{1,2\}, B_{i} \neq b_{i}$ for some $i \in\{1,2\}$ and, moreover, $A_{1}+A_{2}=B_{1}+B_{2}=1$ whenever $(c \delta)$, and $\left(A_{1}+A_{2}\right)\left(B_{1}+\right.$ $\left.B_{2}\right) \neq 1$, whenever $\sim(c \delta)$.

Then the function $f$ satisfies (1) with given $a_{i}, b_{i}$ and defined as above coefficients $A_{i}, B_{i}$ for $i \in\{1,2\}$.

Now, we will provide conditions which guarantee that (1) and (3) are equivalent in the general case. We will say that two non-zero numbers $x$ and $y$ are rationally dependent if there exist rational numbers $\xi$ and $\eta$ such that $\xi x+\eta y=1$.

Before presenting the first main result of this section we prove the following.

Lemma 3. Assume that $a_{i}, b_{i} \in \mathbb{K}^{*}, A_{i}, B_{i}, C_{j} \in \mathbb{K}, i \in\{1,2\}, j \in\{1,2,3,4\}$ and hypothesis ( $\mathrm{H} 5)$ holds.

(i) If (H3) and $a_{1}, a_{2}$ are rationally dependent, then $B_{1}+B_{2}=1$. Conversely, if $B_{1}+B_{2}=1$, then (H3) holds.

(ii) If (H4) and $b_{1}, b_{2}$ are rationally dependent, then $A_{1}+A_{2}=1$. Conversely, if $A_{1}+A_{2}=1$, then ( $\left.\mathrm{H} 4\right)$ holds.

Proof. First we assume that hypothesis (H3) holds and $a_{1}, a_{2}$ are rationally dependent. Indeed, we have

$$
\begin{aligned}
& \Gamma\left(a_{1}\right)=C_{1}+C_{2}=A_{1} B_{1}+A_{1} B_{2}=\Phi\left(a_{1}\right) \Psi\left(b_{1}+b_{2}\right), \\
& \Gamma\left(a_{2}\right)=C_{3}+C_{4}=A_{2} B_{1}+A_{2} B_{2}=\Phi\left(a_{2}\right) \Psi\left(b_{1}+b_{2}\right) .
\end{aligned}
$$


Since $a_{1}, a_{2}$ are non-zero, so are $\Phi\left(a_{1}\right), \Phi\left(a_{2}\right)$ and

$$
\frac{\Gamma\left(a_{1}\right)}{\Phi\left(a_{1}\right)}=\Psi\left(b_{1}+b_{2}\right)=\frac{\Gamma\left(a_{2}\right)}{\Phi\left(a_{2}\right)},
$$

that is $\Gamma\left(a_{i}\right)=\mu \Phi\left(a_{i}\right)$ for some $\mu \neq 0$ and for $i \in\{1,2\}$.

Since $a_{1}, a_{2}$ are rationally dependent, there exist rational numbers $\xi$ and $\eta$ such that $\xi a_{1}+\eta a_{2}=1$ and, consequently (since $\left.\Phi\right|_{\mathbb{Q}}=\left.\Gamma\right|_{\mathbb{Q}}=\mathrm{id}$ ),

$1=\Phi\left(\xi a_{1}+\eta a_{2}\right)=\Gamma\left(\xi a_{1}+\eta a_{2}\right)=\mu \xi \Phi\left(a_{1}\right)+\mu \eta \Phi\left(a_{2}\right)=\mu \Phi\left(\xi a_{1}+\eta a_{2}\right)=\mu$, that is $\mu=1$, and $B_{1}+B_{2}=1$.

For the converse it is enough to observe that $C_{1}+C_{2}=A_{1} B_{1}+A_{1} B_{2}=A_{1}$ and $C_{3}+C_{4}=A_{2} B_{1}+A_{2} B_{2}=A_{2}$, and to define $\Gamma:=\Phi$.

Analogously we show the second item.

Theorem 12. Assume that $a_{i}, b_{i} \in \mathbb{K}^{*}, A_{i}, B_{i}, C_{j} \in \mathbb{K}, i \in\{1,2\}, j \in\{1,2,3,4\}$. If one of the conditions

(I) $(\mathrm{H} 5) \wedge \mathrm{A}_{1}+\mathrm{A}_{2}=1 \wedge \mathrm{B}_{1}+\mathrm{B}_{2}=1$;

(II) $(\mathrm{H} 5) \wedge \mathrm{A}_{1}+\mathrm{A}_{2} \neq 1 \wedge \mathrm{B}_{1}+\mathrm{B}_{2}=1$;

(III) $(\mathrm{H} 5) \wedge \mathrm{A}_{1}+\mathrm{A}_{2}=1 \wedge \mathrm{B}_{1}+\mathrm{B}_{2} \neq 1$;

(IV) $(\mathrm{H} 5) \wedge \mathrm{A}_{1}+\mathrm{A}_{2} \neq 1 \wedge \mathrm{B}_{1}+\mathrm{B}_{2} \neq 1 \wedge\left(\mathrm{A}_{1}+\mathrm{A}_{2}\right)\left(\mathrm{B}_{1}+\mathrm{B}_{2}\right) \neq 1 \wedge$ $\sim(\mathrm{H} 3) \wedge \sim(\mathrm{H} 4)$

(v) $C_{1} C_{4} \neq C_{2} C_{3} \wedge B_{1}+B_{2}=1 \wedge(\mathrm{H} 1) \wedge \sim(\mathrm{H} 2) \wedge(\mathrm{H} 3) \wedge \sim(\mathrm{H} 4)$;

(VI) $C_{1} C_{4} \neq C_{2} C_{3} \wedge A_{1}+A_{2}=1 \wedge \sim(\mathrm{H} 1) \wedge(\mathrm{H} 2) \wedge \sim(\mathrm{H} 3) \wedge(\mathrm{H} 4)$;

(VII) $C_{1} C_{4} \neq C_{2} C_{3} \wedge \sim((\mathrm{H} 1) \vee(\mathrm{H} 2) \vee(\mathrm{H} 3) \vee(\mathrm{H} 4))$

$\wedge\left(C_{1}+C_{2}+C_{3}+C_{4}=A_{1}+A_{2}=B_{1}+B_{2}=1\right.$

$\left.\vee\left(C_{1}+C_{2}+C_{3}+C_{4} \neq 1 \wedge\left(A_{1}+A_{2}\right)\left(B_{1}+B_{2}\right) \neq 1\right)\right)$

holds then (1) and (3) are equivalent.

Proof. First we observe that from Theorem 8 in all first four items there is a biadditive function $g$ satisfying (1) and (3) and we have

$$
A_{1}+A_{2}=1 \wedge B_{1}+B_{2}=1 \Leftrightarrow C_{1}+C_{2}+C_{3}+C_{4}=1 .
$$

In case (I), on account of Lemma 3 the hypotheses (H3) and (H4) hold, so a function $f$ of the form (4), where $g$ is a biadditive function satisfying (5), $\varphi, \psi$ are additive functions satisfying (6) and (7), respectively, and $\delta$ is an arbitrary constant, is a solution of both (3) and (1).

In case (II), on account of Lemma 3 the hypothesis (H4) does not hold. Indeed, if we suppose that (H4) holds, then since $b_{1}+b_{2}=1$, that is, $b_{1}, b_{2}$ are rationally dependent, we would get that $A_{1}+A_{2}=1$, which is a contradiction. The hypothesis (H3) holds, so the general solution of (3) as well as of (1) is $f(x, y)=g(x, y)+\varphi(x)$, where $g$ is a biadditive function satisfying (5) and $\varphi$ is an additive function satisfying (6) as well as (15).

Analogously we show the equivalence of (3) and (1) in cases (III) and (IV). 
Now, we assume that $C_{1} C_{4} \neq C_{2} C_{3}$. On account of Remark 6 , there is no nontrivial biadditive function satisfying (3). This corresponds to the situation when there does not exist an isomorphism $\Phi$ (more exactly, we have $\sim(\mathrm{H} 1)$ ) or there does not exist an isomorphism $\Psi$ (more exactly, we have $\sim(\mathrm{H} 2)$ ), which means that there is no nontrivial biadditive function satisfying (1). It follows also that the general solution of (1) and (3) in this case does not consist of both non-zero $\varphi$ and non-zero $\psi$.

Assumptions $B_{1}+B_{2}=1 \wedge(\mathrm{H} 1) \wedge \sim(\mathrm{H} 2) \wedge(\mathrm{H} 3) \wedge \sim(\mathrm{H} 4)$ ensure that $f(x, y):=\varphi(x)$ with non-zero $\varphi$ such that both (6) and (15) hold true satisfies (1) and (3). Moreover, there is no non-zero $\psi$ such that $f(x, y):=\psi(y)$ satisfies (1) and (3). Further, we have

$$
\Gamma\left(a_{1}+a_{2}\right)=C_{1}+C_{2}+C_{3}+C_{4} .
$$

If $1=A_{1}+A_{2}=\Phi\left(a_{1}+a_{2}\right)$, then $a_{1}+a_{2}=1$, and consequently, $C_{1}+C_{2}+C_{3}+$ $C_{4}=1$, and conversely. That is, either every $\delta$, or only $\delta=0$ simultaneously appears in the solution of both (1) and (3).

Analogously, we treat the case with $A_{1}+A_{2}=1 \wedge \sim(\mathrm{H} 1) \wedge$ $\wedge \sim(\mathrm{H} 3) \wedge(\mathrm{H} 4)$.

From $C_{1} C_{4} \neq C_{2} C_{3} \wedge \sim((\mathrm{H} 1) \vee(\mathrm{H} 2) \vee(\mathrm{H} 3) \vee(\mathrm{H} 4))$ we know that only constant functions are taken into account as solutions of (1) and (3). Condition $C_{1}+C_{2}+C_{3}+C_{4}=A_{1}+A_{2}=B_{1}+B_{2}=1 \vee\left(C_{1}+C_{2}+C_{3}+C_{4} \neq 1\right.$ $\left.\wedge\left(A_{1}+A_{2}\right)\left(B_{1}+B_{2}\right) \neq 1\right)$ ensures that either only the zero function or each constant function is simultaneously a solution of (1) and (3).

The following examples will show that under the hypothesis (H5) we cannot expect that the conjunction ( $\mathrm{H} 1)$ and $B_{1}+B_{2}=1$ is equivalent to (H3) (or that the conjunction (H2) and $A_{1}+A_{2}=1$ is equivalent to (H4)) (cf., Lemma 3 ).

Example 3. Let $a_{1}=\pi, a_{2}=2 \pi, b_{1}=b_{2}=1, A_{1}=e, A_{2}=2 e, B_{1}=B_{2}=$ $1, C_{1}=C_{2}=e, C_{3}=C_{4}=2 e$. Then we have $C_{1}=A_{1} B_{1}, C_{2}=A_{1} B_{2}, C_{3}=$ $A_{2} B_{1}, C_{4}=A_{2} B_{2}$, there exists an isomorphism $\Phi: \mathbb{Q}(\pi) \rightarrow \mathbb{Q}(e)$ such that $\Phi(\pi)=e$ and $\Psi:=$ id. So, we have (H5) and every biadditive function $g: X^{2} \rightarrow$ $Y$ such that

$$
g(\pi x, y)=e g(x, y), \quad x, y \in X
$$

is a solution of both (3) and (1).

Further, there exists $\Gamma: \mathbb{Q}(\pi) \rightarrow \mathbb{Q}(e)$ such that $\Gamma(\pi)=C_{1}+C_{2}=2 e$ and $\Gamma(2 \pi)=C_{3}+C_{4}=4 e$ (i.e., (H3) holds), and there does not exist $\Lambda: \mathbb{Q}\left(b_{1}, b_{2}\right)=$ $\mathbb{Q} \rightarrow \mathbb{Q}\left(C_{1}+C_{3}, C_{2}+C_{4}\right)=\mathbb{Q}(e)$ such that $\Lambda(1)=3 e$. Consequently, the function $f(x, y)=g(x, y)+\varphi(x), x, y \in X$, with additive $\varphi$ satisfying the condition

$$
\varphi(\pi x)=2 e \varphi(x), \quad x \in X,
$$

is the general solution of (3). 
Since $A_{1}+A_{2} \neq 1$ and $B_{1}+B_{2} \neq 1$, the function $f=g$ with biadditive $g$ satisfying (26) is the general solution of (1), so (1) and (3) are not equivalent.

Consequently, we see that in Lemma 3 we have to make an additional assumption to (H3) in order to get $B_{1}+B_{2}=1$. The question is how weak this additional assumption may be.

We say further that $x$ and $y$ are algebraically independent over $\mathbb{Q}$ if they do not satisfy any non-trivial polynomial equation with coefficients in $\mathbb{Q}$. It is interesting to ask whether rational dependence in Lemma 3 can be exchanged for algebraic dependence. The answer is given in the next example.

Example 4. Let $a_{1}=\sqrt{\pi}, a_{2}=2 \pi+1, A_{1}=e, A_{2}=2 e^{2}+1$ and let $b_{1}, b_{2}, B_{1}, B_{2}$ and $C_{j}, j \in\{1,2,3,4\}$, be such that (H5) and (H3) are satisfied. We have $\Phi(\sqrt{\pi})=e$ and

$$
\begin{aligned}
& \Gamma(\sqrt{\pi})=C_{1}+C_{2}=A_{1}\left(B_{1}+B_{2}\right)=e\left(B_{1}+B_{2}\right), \\
& \Gamma(2 \pi+1)=C_{3}+C_{4}=A_{2}\left(B_{1}+B_{2}\right)=\left(2 e^{2}+1\right)\left(B_{1}+B_{2}\right) .
\end{aligned}
$$

From the other side,

$$
\Gamma(2 \pi+1)=2 \Gamma(\sqrt{\pi})^{2}+1=2 e^{2}\left(B_{1}+B_{2}\right)^{2}+1 .
$$

If $\mu:=B_{1}+B_{2}$, then $\left(2 e^{2}+1\right) \mu=2 e^{2} \mu^{2}+1$, that is $2 e^{2} \mu^{2}-\left(2 e^{2}+1\right) \mu+1=0$. Solving this equation with respect to $\mu$, we obtain $\mu_{1}=\frac{1}{2 e^{2}}$ and $\mu_{2}=1$, so in this case $B_{1}+B_{2}$ may be different from 1 . Therefore in Lemma 3 we cannot replace rational dependence by algebraic dependence.

Later on we will still need the following result.

Lemma 4. Let $a_{i}, b_{i}, C_{j} \in \mathbb{K}^{*}, i \in\{1,2\}, j \in\{1,2,3,4\}$ and $C_{1} C_{4}=C_{2} C_{3}$. If $a_{1}, a_{2}$ or $b_{1}, b_{2}$ are rationally dependent, then each non-zero biadditive function satisfying (3) satisfies also (1) with

$$
B_{1}=\xi C_{1}+\eta C_{3}, \quad B_{2}=\frac{B_{1} C_{2}}{C_{1}}, \quad A_{i}=\frac{C_{2 i-1}}{B_{1}}, i \in\{1,2\}
$$

whenever $\xi a_{1}+\eta a_{2}=1$ for some $\xi, \eta \in \mathbb{Q}$, and

$$
A_{1}=\mu C_{1}+\nu C_{2}, \quad A_{2}=\frac{A_{1} C_{3}}{C_{1}}, \quad B_{i}=\frac{C_{i}}{A_{1}}, i \in\{1,2\}
$$

whenever $\mu b_{1}+\nu b_{2}=1$ for some $\mu, \nu \in \mathbb{Q}$.

Proof. We assume that $a_{1}, a_{2}$ are rationally dependent and $g$ is a biadditive function satisfying (3). Then there exist two rational constants $\xi, \eta$ such that $\xi a_{1}+\eta a_{2}=1$ and

$$
\begin{aligned}
g\left(x, b_{1} y\right) & =g\left(\left(\xi a_{1}+\eta a_{2}\right) x, b_{1} y\right)=g\left(\xi a_{1} x, b_{1} y\right)+g\left(\eta a_{2} x, b_{1} y\right) \\
& =\xi g\left(a_{1} x, b_{1} y\right)+\eta g\left(a_{2} x, b_{1} y\right)=\left(\xi C_{1}+\eta C_{3}\right) g(x, y),
\end{aligned}
$$


so,

$$
B_{1}=\xi C_{1}+\eta C_{3},
$$

and the further coefficients $A_{1}, A_{2}, B_{2}$ are already uniquely determined (cf., Remarks 7 and 8), $A_{i}:=\frac{C_{2 i-1}}{B_{1}}, i \in\{1,2\}$ and $B_{2}:=\frac{B_{1} C_{2}}{C_{1}}$, moreover

$$
g\left(a_{i} x, y\right)=A_{i} g(x, y), i \in\{1,2\} \quad \text { and } \quad g\left(x, b_{2} y\right)=B_{2} g(x, y) .
$$

Analogously, we proceed in the case when $b_{1}, b_{2}$ are rationally dependent.

The next result discusses the alienation problem.

Theorem 13. Assume that $a_{i}, b_{i} \in \mathbb{K}^{*}, C_{j} \in \mathbb{K}, i \in\{1,2\}, j \in\{1,2,3,4\}$ and $f: X^{2} \rightarrow Y$ satisfies (3). Define $A_{i}, B_{i} \in \mathbb{K}, i \in\{1,2\}$ :

(I) if $C_{1} C_{4}=C_{2} C_{3}, a_{1}+a_{2}=b_{1}+b_{2}=1$, (H3) and (H4), then take $A_{1}:=C_{1}+C_{2}, A_{2}:=C_{3}+C_{4}, B_{1}:=C_{1}+C_{3}$ and $B_{2}:=C_{2}+C_{4}$;

(II) if $C_{1} C_{4}=C_{2} C_{3}, a_{1}+a_{2} \neq 1, b_{1}+b_{2}=1$, (H3) and (H2) with $B_{i}:=$ $\frac{C_{i}}{C_{1}+C_{2}}, i \in\{1,2\}$, then take $A_{1}:=C_{1}+C_{2}, A_{2}:=C_{3}+C_{4}$, and $B_{i}, i \in\{1,2\}$, as defined above;

(III) if $C_{1} C_{4}=C_{2} C_{3}, a_{1}+a_{2}=1, b_{1}+b_{2} \neq 1$ and (H4) and (H1) with $A_{i}:=\frac{C_{2 i-1}}{C_{1}+C_{3}}, i \in\{1,2\}$, then take $B_{1}:=C_{1}+C_{3}, B_{2}:=C_{2}+C_{4}$, and $A_{i}, i \in\{1,2\}$ as defined above;

(IV) if $C_{1} C_{4}=C_{2} C_{3}, a_{1}+a_{2} \neq 1, b_{1}+b_{2} \neq 1, C_{1}+C_{2}+C_{3}+C_{4} \neq 1$, and $\left(\left(a_{1}, a_{2}\right.\right.$ are rationally dependent $\wedge \sim(\mathrm{H} 4) \wedge(\mathrm{H} 1) \wedge(\mathrm{H} 2)$ with (27) $) \vee\left(b_{1}, b_{2}\right.$ are rationally dependent $\wedge \sim(\mathrm{H} 3) \wedge(\mathrm{H} 1) \wedge(\mathrm{H} 2)$ with (28))), then take $A_{1}, A_{2}, B_{1}, B_{2}$ as indicated above;

(v) if $C_{1} C_{4} \neq C_{2} C_{3} \wedge(\mathrm{H} 3) \wedge \sim(\mathrm{H} 4)$, then take $A_{1}:=C_{1}+C_{2}, A_{2}:=$ $C_{3}+C_{4}$, and arbitrary $B_{1}, B_{2}$ such that $\sim(\mathrm{H} 2)$ and $B_{1}+B_{2}=1$;

(vI) if $C_{1} C_{4} \neq C_{2} C_{3} \wedge \sim(\mathrm{H} 3) \wedge(\mathrm{H} 4)$, then take $B_{1}:=C_{1}+C_{3}, B_{2}:=$ $C_{2}+C_{4}$, and arbitrary $A_{1}, A_{2}$ such that $\sim(\mathrm{H} 1)$ and $A_{1}+A_{2}=1$;

(VII) if $C_{1} C_{4} \neq C_{2} C_{3} \wedge \sim(\mathrm{H} 3) \wedge \sim(\mathrm{H} 4)$, then take arbitrary $A_{1}, A_{2}, B_{1}, B_{2}$ such that $\sim(\mathrm{H} 1) \wedge \sim(\mathrm{H} 2)$ and, moreover, $A_{1}+A_{2}=B_{1}+B_{2}=1$ whenever $C_{1}+C_{2}+C_{3}+C_{4}=1$, and $\left(A_{1}+A_{2}\right)\left(B_{1}+B_{2}\right) \neq 1$, whenever $C_{1}+C_{2}+C_{3}+C_{4} \neq 1$.

Then $f$ satisfies (1) with given $a_{i}, b_{i}$ and with coefficients $A_{i}, B_{i}$ for $i \in\{1,2\}$ as defined above.

Proof. In case (I), since $C_{1} C_{4}=C_{2} C_{3}$, we have

$$
A_{1} B_{1}=\left(C_{1}+C_{2}\right)\left(C_{1}+C_{3}\right)=C_{1}\left(C_{1}+C_{2}+C_{3}+C_{4}\right)=C_{1},
$$

and analogously we check that $C_{2}=A_{1} B_{2}, C_{3}=A_{2} B_{1}, C_{4}=A_{2} B_{2}$. Hypotheses (H1) and (H2) hold with $\Phi:=\Gamma$ and $\Psi:=\Lambda$. Moreover, it is easy to see that $A_{1}+A_{2}=B_{1}+B_{2}=1$ and the assertion follows from Theorem 12 .

For (II), it is necessary first to observe that $C_{1}+C_{2} \neq 0$. Indeed, by (H3), there exists the isomorphism $\Gamma$ such that (among others) $\Gamma\left(a_{1}\right)=C_{1}+C_{2}$, and since $a_{1} \neq 0$, so does $C_{1}+C_{2}$. The rest follows from Theorem 12 . 
We treat cases (III)-(VII) similarly (using additionally Lemma 4 for proving (IV)) .

Remark 10. In the above theorem, for alienation in case (v) it is enough to take $B_{1}:=q \in \mathbb{Q} \backslash\left\{b_{1}, 0,1\right\}, B_{2}:=1-B_{1}$, in case (VI) it is enough to take $A_{1}:=q \in \mathbb{Q} \backslash\left\{a_{1}, 0,1\right\}, A_{2}:=1-A_{1}$, and in case (VII), $A_{1}:=C_{1}+C_{2}, A_{2}:=$ $C_{3}+C_{4}, B_{1}:=C_{1}+C_{3}$ and $B_{2}:=C_{2}+C_{4}$.

In case (IV) of Theorem 13 we can not replace rational dependence with algebraic dependence, which is shown by the following.

Example 5. Let $a_{1}=\sqrt{\pi}, a_{2}=2 \pi+1, b_{1}=\frac{1}{20}, b_{2}=\frac{1}{2 e^{2}}-\frac{1}{20}, C_{1}=\frac{e}{20}, C_{2}=$ $\frac{1}{2 e}-\frac{e}{20}, C_{3}=\frac{2 e^{2}+1}{20}, C_{4}=1+\frac{1}{2 e^{2}}-\frac{2 e^{2}+1}{20}$. We have to take $B_{1}=\frac{1}{20}$ (we want (H2) to hold) and $A_{1}=e, A_{2}=2 e^{2}+1, B_{2}=\frac{1}{2 e^{2}}-\frac{1}{20}$. Then, it is easy to check that the condition

$$
\begin{aligned}
& C_{1} C_{4}=C_{2} C_{3} \wedge a_{1}+a_{2} \neq 1 \wedge b_{1}+b_{2} \neq 1 \wedge C_{1}+C_{2}+C_{3}+C_{4} \neq 1 \\
& \wedge\left(\left(a_{1}, a_{2} \text { are algebraic dependent } \wedge \sim(\mathrm{H} 4) \wedge(\mathrm{H} 1) \wedge(\mathrm{H} 2)\right)\right.
\end{aligned}
$$

is satisfied, moreover (H3) holds. The additive function $\varphi$ such that $\varphi(\sqrt{\pi} x)=$ $e \varphi(x)$ is a solution of (3) but is not a solution of (1).

Theorem 12 gives sufficient conditions for the equivalence of (1) and (3). Therefore, it seems natural to state the following.

Problem 2. Find conditions which completely describe the equivalence between (1) and (3).

Open Access. This article is licensed under a Creative Commons Attribution 4.0 International License, which permits use, sharing, adaptation, distribution and reproduction in any medium or format, as long as you give appropriate credit to the original author(s) and the source, provide a link to the Creative Commons licence, and indicate if changes were made. The images or other third party material in this article are included in the article's Creative Commons licence, unless indicated otherwise in a credit line to the material. If material is not included in the article's Creative Commons licence and your intended use is not permitted by statutory regulation or exceeds the permitted use, you will need to obtain permission directly from the copyright holder. To view a copy of this licence, visit http:// creativecommons.org/licenses/by/4.0/.

Publisher's Note Springer Nature remains neutral with regard to jurisdictional claims in published maps and institutional affiliations.

\section{References}

[1] Aczél, J.: Über eine Klasse von Funktionalgleichungen. Comment. Math. Helv. 21, 247$252(1948)$

[2] Aczél, J.: Lectures on Functional Equations and Their Applications. Academic Press, New York-London (1966) 
[3] Aczél, J., Dhombres, J.: Functional Equations in Several Variables. Encyclopedia of Mathematics and its Applications, 31. Cambridge University Press, Cambridge (1989)

[4] Bahyrycz, A., Olko, J.: On stability of the general linear equation. Aequat. Math. 89, 1461-1474 (2015)

[5] Ciepliński, K.: On a functional equation connected with be-linear mappings and its Hyers-Ulam stability. J. Nonlinear Sci. Appl. 10, 5914-5921 (2017)

[6] Daróczy, Z.: Notwendige und hinreichende Bedingungen für die Existenz von nichtkonstanten Lösungen linearer Funktionalgleichungen. Acta Sci. Math. Szeged 22, 31-41 (1961)

[7] Dhombres, J.: Relations de dépendance entre les équat fonctionnelles de Cauchy. Aequat. Math. 35, 186-212 (1988)

[8] Ger, R., Sablik, M.: Alien functional equations: a selective survey of results. Developments in functional equations and related topics, 107-147, Springer Optim. Appl., 124, Springer, Cham (2017)

[9] Gselmann, E., Kiss, G., Vincze, Cs.: On a class of linear functional equations without range condition. Aequat. Math. 94, 473-509 (2020)

[10] Kuczma, M.: An Introduction to the Theory of Functional Equations and Inequalities. Cauchy's Equation and Jensen's Inequality. Second edition. Attila Gilányi (ed.) Birkhäuser Verlag, Basel (2009)

Anna Bahyrycz

AGH University of Science and Technology

Faculty of Applied Mathematics

Mickiewicza 30

30-059 Kraków

Poland

e-mail: bahyrycz@agh.edu.pl

Justyna Sikorska

Faculty of Science and Technology

University of Silesia in Katowice

Bankowa 14

40-007 Katowice

Poland

e-mail: justyna.sikorska@us.edu.pl

Received: November 3, 2020

Revised: May 26, 2021

Accepted: May 27, 2021 